\title{
Type 2 diabetes-associated carotid plaque burden is increased in patients with retinopathy compared to those without retinopathy
}

\author{
Núria Alonso ${ }^{1 \dagger}$, Alicia Traveset $^{2 \dagger}$, Esther Rubinat ${ }^{3,4}$, Emilio Ortega $^{5}$, Nuria Alcubierre $^{4}$, Jordi Sanahuja ${ }^{7}$,
} Marta Hernández ${ }^{3,6}$, Angels Betriu $^{6}$, Carmen Jurjo², Elvira Fernández ${ }^{6,8}$ and Didac Mauricio ${ }^{{ }^{*}}$

\begin{abstract}
Background: Cardiovascular disease (CVD) is the leading cause of mortality among subjects with type 2 diabetes (T2D), and diabetic retinopathy (DR) has been associated with an increased risk for CVD. The present study was designed to test the concept that T2D patients with DR, but without previous cardiovascular (CV) events and with normal renal function, have an increased atherosclerotic burden compared with patients without DR.

Methods: A cross-sectional study was performed using patients with normal renal function (estimated glomerular filtration rate (eGFR) $>60 \mathrm{ml} / \mathrm{min}$ ) and without previous CV events. A total of 312 patients (men, 51\%; mean age, $57 \mathrm{yrs}$; age range 40-75 yrs) were included in the study; 153 (49\%) of the patients had DR. B-mode carotid ultrasound imaging was performed for all of the study subjects to measure the carotid intima-media thickness (CIMT) and carotid plaques in the common carotid artery (CCA), bifurcation and internal carotid artery (ICA).

Results: The percentage of carotid plaques in T2D patients with DR was higher than in T2D patients without DR ( $68 \%$ vs. $52.2 \%, p=0.0045$ ), and patients with DR had a higher prevalence of $\geq 2$ carotid plaques ( $44.4 \%$ vs. $21.4 \%$; $p<0.0001$ ). No differences were observed in the cIMT measured at different carotid regions between the patients with or without DR. Using multivariate logistic regression (adjustment for major risk factors for atherosclerosis), DR was independently associated with mean-internal $\mathrm{CIMT}(p=0.0176)$, with the presence of carotid plaques $(p=0.0366)$ and with carotid plaque burden ( $\geq 2$ plaques; $p<0.0001$ ).

Conclusions: The present study shows that DR in T2D patients without CVD and with normal renal function is associated with a higher atherosclerotic burden (presence and number of plaques) in the carotid arteries. These patients may be at a higher risk for future CV events; therefore, an ultrasound examination of the carotid arteries should be considered in patients with DR for more careful and individualised CV assessment and follow-up.
\end{abstract}

Keywords: Type 2 diabetes, Cardiovascular disease, Retinopathy, Carotid plaque

\section{Background}

The deleterious effects of hyperglycaemia are classically separated into microvascular (retinopathy, diabetic nephropathy and neuropathy) and macrovascular complications (coronary artery disease, peripheral arterial disease and cerebrovascular disease).

\footnotetext{
* Correspondence: didacmauricio@gmail.com

${ }^{\dagger}$ Equal contributors

'Department of Endocrinology and Nutrition, Health Sciences Research Institute and University Hospital Germans Trias i Pujol, Badalona, Spain Full list of author information is available at the end of the article
}

Traditionally, the micro- and macrovascular complications of diabetes have been viewed, studied, and treated as distinct and independent disorders. However, accumulating data from epidemiological and pathophysiological studies suggest that these vascular complications may share common pathophysiological mechanisms beyond those related to traditional cardiovascular $(\mathrm{CV})$ risk factors. Data obtained from epidemiological studies have clearly demonstrated that diabetic retinopathy (DR), a common chronic microvascular complication of diabetes, is associated with macrovascular disease [1] as

\section{() BioMed Central}

(c) 2015 Alonso et al.; licensee BioMed Central. This is an Open Access article distributed under the terms of the Creative Commons Attribution License (http://creativecommons.org/licenses/by/4.0), which permits unrestricted use, distribution, and reproduction in any medium, provided the original work is properly credited. The Creative Commons Public Domain Dedication waiver (http://creativecommons.org/publicdomain/zero/1.0/) applies to the data made available in this article unless otherwise stated. 
well as with increased CV morbidity and mortality in patients with type 2 diabetes mellitus (T2D) [2]. In these patients, the presence of DR has been described as an independent risk factor for incident coronary heart disease $[3,4]$ and ischaemic stroke [5]. Among the possible mechanisms described to explain the interconnection between diabetic micro- and macroangiopathy are metabolic disturbances [6]. Other authors have suggested that microangiopathy of the vasa vasorum (VV), the plexus of microvessels that partially provides oxygen and nutrients to the walls of large arteries, may be involved in diabetic atherosclerosis $[7,8]$. Our group recently reported that T2D patients with DR had increased angiogenesis of the $\mathrm{VV}$ of the common carotid artery (CCA) [9]. These findings were recently confirmed by Sampson et al. in a larger study of T2D patients [10].

When a clinical CV event occurs, atherosclerotic disease is difficult to reverse. In these cases, ultrasonography of the carotid arteries is frequently used to detect early signs of atherosclerosis, i.e., increased thickness of the arterial wall or the occurrence of plaques. Ample data suggest that plaque and carotid intima-media thickness (cIMT) are associated with prevalent and incident coronary heart disease (CHD) and stroke, with the presence of plaque generally having a stronger association with CVD compared to cIMT alone [11]. Thus, it has recently been demonstrated that ultrasound assessment of a carotid plaque and its total volume or total area progression is a stronger predictor of future $\mathrm{CV}$ events than cIMT measurement [12,13]. A high prevalence (between $43 \%$ and $64 \%$ ) of carotid plaques has been described in T2D patients without evidence of $\mathrm{CV}$ disease (CVD) [14-16]. Prospective studies conducted in T2D patients free of any $\mathrm{CV}$ event have shown that the percentage of patients with carotid artery plaques is higher in those who develop a CV event compared with those who are free from CV events $[15,16]$. Recent epidemiological studies have demonstrated that chronic kidney disease (CKD), defined as an estimated glomerular filtration rate (eGFR) $<60 \mathrm{ml} / \mathrm{min}$, is an independent risk factor for atherosclerotic disease [17] and for the presence of carotid plaques [18]. In T2D patients, a high prevalence of low eGFR without associated albuminuria has been reported and described to be associated with atherosclerosis of peripheral arteries, independently of albuminuria, [19] and with cIMT in several [20], but not all, studies [21,22].

To test the concept that patients with T2D and DR have an increased prevalence of subclinical atherosclerosis, as shown using carotid plaque burden and cIMT measurements, we investigated the association between these measures and retinopathy in a group of patients with T2D but without previous CVD and with normal renal function.

\section{Methods}

\section{Subjects}

T2D patients were selected to participate in this singlecentre study, which aimed to investigate the association between carotid atherosclerosis and DR in T2D. All study participants were recruited from the outpatient clinic at our centre, and potential candidates were identified by screening patients enrolled in the diabetic eye disease programme of our centre as well as those visiting the diabetic retinopathy unit. The inclusion criteria for both groups were as follows: age range, 40-75 yrs; absence of established impaired renal function (calculated glomerular filtration rate (eGFR) $<60 \mathrm{ml} / \mathrm{min}$ ); and known CVD. From the total number of subjects who were initially recruited, 60 subjects were excluded after the screening visit because of the presence of an exclusion criterion (previous CVD, impaired renal function, increased urine albumin excretion, or not having T2D), and 15 additional patients withdrew from the study after the initial screening visit (consented withdrawal before completing the study assessment). A total of 312 participants with $(n=153)$ or without $(n=159)$ DR were included in the study. To achieve a uniform age distribution, patients with and without DR were selected to have representative and similar numbers of patients by gender and age (stratified according to 5-yr age intervals). However, we could not recruit enough patients with retinopathy in the age range between 40 and 50 yrs. For the purpose of this study, a patient was arbitrarily considered to have previous hypertension or dyslipidaemia if the patient was taking medication for the given condition. The weight, height and waist circumference of the subjects were measured using standardised methods, and the blood pressure (mean of 2 measurements, $5 \mathrm{~min}$ apart) of the subjects was measured after $10 \mathrm{~min}$ in the seated position using a blood pressure monitor (HEM7001E, Omron, Barcelona, Spain). The patients underwent a complete eye evaluation by experienced ophthalmologists (AT and CJ) to assess the presence or absence of diabetic retinopathy according to an international clinical DR consensus [23]. Retinopathy was evaluated using multifield stereoscopic retinal photography with the following definitions: a) mild nonproliferative DR microaneurysms only; b) moderate nonproliferative DR - more than just microaneurysms but less than severe nonproliferative DR; c) severe nonproliferative DR by any of the following - more than 20 intraretinal haemorrhages in each of 4 quadrants, definite venous beading in 2+ quadrants, prominent intraretinal microvascular abnormalities in 1+ quadrant, and no signs of proliferative retinopathy; and d) proliferative DR, by neovascularisation and/or vitreous/preretinal haemorrhage. In addition to the anamnestic evaluation and physical examination, the patients' clinical records were reviewed to rule out 
any previously known $\mathrm{CV}$ events or associated revascularisation procedures, including coronary heart disease, cerebrovascular disease, or peripheral vascular disease (including the diagnosis of diabetic foot disease). Ours is the only reference hospital for CVDs and procedures in the region, but we also had access to reports from three small hospitals in the region. Therefore, along with the anamnestic evaluation, we could ascertain any $\mathrm{CV}$ events that occurred in the health-care area. Heart failure and macroalbuminuria (urine albumin/creatinine ratio $>299 \mathrm{mg} / \mathrm{g}$ ) were also considered exclusion criteria. Serum and spot urine samples were collected in the fasting state, and all serum and urine tests were performed using standard laboratory methods. Low-density lipoprotein cholesterol was estimated using the Friedewald formula, and eGFR was estimated using the diet modification in renal disease (MDRD-4) formula [24]. Haemoglobin $\mathrm{A}_{1 \mathrm{c}}\left(\mathrm{HbA}_{1 \mathrm{c}}\right)$ levels were determined using HPLC (Variant ${ }^{\mathrm{TM}}$, Bio-Rad Laboratories SA, Spain), and its concentrations are expressed in National Glycohemoglobin Standardization Program/Diabetes Control and Complications Trial units. Urine albumin was measured using an immunoturbidimetric method and a Roche/Hitachi Modular P analyser (Roche Diagnostics, Spain).

\section{Carotid ultrasound imaging study}

All of the study participants underwent the same carotid ultrasound protocol. All measures and ultrasound studies were assessed by the same researcher (ER), who was blinded to the conditions of the participants and did not have access to the study data. B-mode ultrasound imaging was performed using a Siemens Sequoia 512 and a $15-\mathrm{Mhz}$ linear array probe. Moreover, a standardised imaging protocol was performed to evaluate intimamedia-adventitia thickness (IMT), defined as the distance between the lumen-intima and the media-adventitia ultrasound interfaces (intima-media complex), and plaque presence in the carotid arteries. The patients were examined from their back; they were placed in the supine position with the head turned $45^{\circ}$ contralateral to the side of scanning. Images were obtained in longitudinal sections, with a single lateral angle of insonation and optimisation of the image to the far wall. The B-mode images of the left and right segments were recorded and electronically stored. The last (previous to the bulb) and first (starting at the flow divider) centimetres of the common and internal carotid arteries, respectively, and the total length of the bulb were used for IMT measurements, which were performed off-line using semiautomatic software. The data (mean IMT and mean-maximum IMT) from each segment were provided, and the rightand left-side values were averaged to obtain the mean and mean-maximum common carotid (CC), carotid bulb, and internal carotid (ICA) measurements. Plaques were identified using B-mode and colour Doppler examinations in both the longitudinal and transverse planes to consider circumferential asymmetry and were defined as a "focal structure that encroaches into the arterial lumen of at least $0.5 \mathrm{~mm}$ or $50 \%$ of the surrounding cIMT value or demonstrates a thickness of $1.5 \mathrm{~mm}$, as measured from the media adventitia interference to the intima-lumen surface" according to the Mannheim consensus [25].

The local Ethics Committee of Hospital Arnau de Vilanova (Lleida, Spain) approved the protocol, and all of the patients signed written informed consent forms.

\section{Sample size}

To determine the sample size, we used preliminary data from a previous study of our group in which the plaque frequency was $69 \%$ in patients with retinopathy or $52 \%$ in patients without retinopathy [10]. We calculated a sample size of 126 subjects for each study group, which would allow an $80 \%$ power to detect differences between groups with a significance level of $<0.05$. Thus, the number of patients included in each group was sufficient to test the initial hypothesis.

\section{Statistical analysis}

The data are presented as the median values and 25th and 75th percentiles, means \pm standard deviations (SDs), and $\mathrm{n}(\%)$, as appropriate. Non-normally distributed variables were log transformed to reduce skewness, and normality was re-evaluated. Between-group (DR vs. nonDR, male vs. female, and with hypertension vs. without hypertension, or dyslipidaemia, or smoking habit) differences in anthropometric, clinical, cIMT, and laboratory variables were evaluated using the chi-squared test (for categorical variables), Wilcoxon test (for continuous, nonnormally distributed variables), or Student's $t$-test (for continuous, normally distributed variables). Unadjusted and adjusted general linear models (PROC GLM in SAS) were used to test whether cIMT values differed between diabetic patients with retinopathy vs. those without retinopathy. Univariate or multiple logistic regression models were used to evaluate the differences in plaque presence between the groups. The following factors were entered into the adjusted general linear and multiple logistic regression models: age, sex, body mass index, presence of hypertension, presence of dyslipidaemia, smoking habit, urinary albumin excretion rate and eGFR. The significance level was defined as $p \leq 0.05$, and all analyses were performed using SAS software, v. 9.2 (SAS Institute Inc., USA).

\section{Results}

Clinical variables

Among the 312 study patients with T2D, 153 (49.03\%)

had DR. The patients with retinopathy were older due to 
difficulties recruiting patients with DR who were aged $<50$ yrs. As typically observed in previous studies comparing patients with and without DR, the patients with this condition had longer diabetes duration and were more likely to receive insulin treatment and antiplatelet agents. These patients also exhibited a higher prevalence of hypertension, higher systolic blood pressure, and increased waist circumference, HbA1c level, HDL-c level, and urinary albumin/creatinine ratio compared with those without DR (Table 1). The distribution of retinopathy grades was as follows: 60 patients had mild retinopathy (39.2\%), 57 patients had moderate retinopathy (37.3\%), 29 patients had severe nonproliferative retinopathy (18.9\%), and 7 patients had severe proliferative retinopathy $(4.6 \%)$.

\section{Ultrasound examination}

The mean and mean-maximum cIMT at different territories (common, bulb, and internal carotid) did not differ between the patients with DR and those without DR, except that a higher mean-maximum common cIMT was observed in the patients with DR $(p=0.0187)$; these differences disappeared after adjustment (Table 2). The general linear models were used to evaluate the cIMT determinants. Age $(p<0.0001)$ and active smoking $(p=0.0641)$; age $(p=0.0154)$ and hypertension $(p=0.0140)$; and age $(p=0.0131)$, sex $(p=0.0327)$, and the presence of retinopathy $(p=0.0176)$ were independently associated with mean CCA-IMT, bulb-IMT, and ICA-IMT, respectively. No association was found between any of the cIMT measurements at different territories or between the UAE and eGFR values or diabetes duration.

The percentage of patients with carotid plaques was higher in those with DR compared to those without DR (68\% vs. $52.2 \%, \mathrm{p}=0.0045)$, and of these patients, the DR group contained more patients with $\geq 2$ carotid plaques $(44.4 \%$ vs. $21.4 \%, p<0.0001)$. No association was found between carotid plaque burden ( $\geq 2$ carotid plaques) and the duration of diabetes. In relation to the territory, there was a tendency towards a higher frequency of plaques at the internal carotid artery (ICA) in the group of patients with DR ( $36 \%$ vs. $49 \%, \mathrm{p}=0.07)$. The variables that were independently associated with the presence of carotid plaques were age $(p=0.0022)$, hypertension $(p=0.0300)$, the presence of retinopathy $(\mathrm{p}=0.0366)$, and active smoking $(\mathrm{p}=0.0109)$ (Table 3$)$. The variables that were associated with the presence of $\geq 2$ carotid plaques were age $(\mathrm{p}<0.0001)$, sex $(\mathrm{p}=$ $0.0015)$, dyslipidaemia $(\mathrm{p}=0.0031)$, and the presence of retinopathy $(\mathrm{p}<0.0001)$ (Table 4$)$.

Table 1 Characteristics of the study groups

\begin{tabular}{|c|c|c|c|}
\hline & $\begin{array}{l}\text { Diabetes without } \\
\text { retinopathy }(n=159)\end{array}$ & $\begin{array}{l}\text { Diabetes with } \\
\text { retinopathy }(n=153)\end{array}$ & $p$ \\
\hline Sex (male/female) & $82 / 77$ & $76 / 77$ & 0.7373 \\
\hline Non-Caucasian & $7(4.4 \%)$ & $6(4.6 \%)$ & 0.9413 \\
\hline Age (yr) & $59(48-66)$ & $61(54-68)$ & 0.0127 \\
\hline Disease duration (yr) & $6(2-10)$ & $11(6-20)$ & $<0.0001$ \\
\hline Insulin treatment $(\mathrm{n})$ & $20(12.6 \%)$ & $84(54.9 \%)$ & $<0.0001$ \\
\hline Smoking (current/past/never) & $71 / 54 / 32$ & $78 / 44 / 30$ & 0.5134 \\
\hline Antiplatelet agents & $112 / 47(29.6 \%)$ & $82 / 71(46.4 \%)$ & 0.0022 \\
\hline Dyslipidaemia (yes/no) & $88 / 71(44.7 \%)$ & $79 / 74(48.4 \%)$ & 0.5111 \\
\hline Hypertension (yes/no) & $83 / 76(47.8 \%)$ & 48/105 (68.3\%) & 0.0002 \\
\hline Systolic BP (mm Hg) & $134(123-145)$ & $143(133-159)$ & $<0.0001$ \\
\hline Diastolic BP (mm Hg) & $76(70-83)$ & $77.5(68.5-85.5)$ & 0.7526 \\
\hline $\mathrm{HR}(\mathrm{b} / \mathrm{min})$ & $75(68-82)$ & $77(70-86)$ & 0.0767 \\
\hline Body mass index $\left(\mathrm{kg} / \mathrm{m}^{2}\right)$ & $30.3(27.4-34.0)$ & $31.1(28.3-35)$ & 0.2477 \\
\hline Waist circumference (cm) & $103(96-111)$ & $107.5(163-114)$ & 0.0039 \\
\hline Haemoglobin $A_{1 c}(\%)$ & $7.1(6.5-7.9)$ & $8.1(7.2-9.1)$ & $<0.0001$ \\
\hline Total cholesterol (mmol/l) & $184(163-207)$ & $181(162-204.5)$ & 0.9889 \\
\hline HDL-cholesterol (mmol/l) & $47(40-57)$ & $50.5(42-60.5)$ & 0.0308 \\
\hline LDL-cholesterol (mmol/l) & $108(90.2-130.2)$ & $105.4(86.5-127.8)$ & 0.2694 \\
\hline Triglycerides $(\mathrm{mmol} / \mathrm{l})$ & $118(89-171)$ & $116(83-168)$ & 0.6181 \\
\hline Serum creatinine $(\mu \mathrm{mol} / \mathrm{l})$ & $0.79(0.69-0.92)$ & $0.79(0.68-0.93)$ & 0.7975 \\
\hline Urinary albumin/creatinine ratio, mg/g & $5.8(3.2-11)$ & $12.4(6-32.7)$ & $<0.0001$ \\
\hline
\end{tabular}

Data are presented as the median values and interquartile ranges. BP, blood pressure. 
Table 2 Results of the ultrasonographic carotid study: carotid intima-media thickness and presence of carotid plaques

\begin{tabular}{llll}
\hline & $\begin{array}{l}\text { No retinopathy } \\
(\mathbf{n = 1 5 9 )}\end{array}$ & $\begin{array}{l}\text { Retinopathy } \\
(\mathbf{n}=153)\end{array}$ & P value \\
\hline Mean common cIMT & $0.789(0.686-0.889)$ & $0.802(0.731-0.907)$ & 0.1474 \\
Mean-max common cIMT & $0.941(0.820-1.049)$ & $0.986(0.889-1.073)$ & 0.0187 \\
Mean bulb cIMT & $0.835(0759-0.955)$ & $0.861(0.723-0.928)$ & 0.6674 \\
Mean-max bulb & $1.047(0.922-1.199)$ & $1.007(0.919-1.227)$ & 0.7470 \\
Mean-internal cIMT & $0.677(0-566-0.782)$ & $0.623(0.534-0.713)$ & 0.1151 \\
Mean-max internal cIMT & $0.845(0.704-1.009)$ & $0.810(0.685-0.909)$ & 0.2070 \\
Carotid plaques, $\mathrm{n}(\%)$ & $83(52.2 \%)$ & $104(68 \%)$ & 0.0045 \\
Carotid plaques (none/1/>1) (n) & $76 / 49 / 34$ & $49 / 36 / 68$ & $<0.0001$ \\
Common carotid plaque, $\mathrm{n}(\%)$ & $9(10.8 \%)$ & $19(18.3 \%)$ & 0.1574 \\
Bulb plaque, $\mathrm{n}(\%)$ & $67(80.7 \%)$ & $89(85.6 \%)$ & 0.3752 \\
Internal carotid plaque, $\mathrm{n}(\%)$ & $30(36.1 \%)$ & $51(49 \%)$ & 0.0771 \\
\hline
\end{tabular}

No differences in cIMT measurements or in plaque presence were observed among the groups with different grades of retinopathy (mild vs. moderate/severe/proliferative).

\section{Discussion}

The present study shows that T2D patients with DR who are free of clinical CVD and with normal renal function have increased atherosclerotic burden in their carotid arteries compared with patients without DR.

Epidemiological studies have demonstrated that DR in T2D patients is associated with a 1.7-fold increased risk of CV events, such as stroke, coronary artery disease and heart failure [26]. Notably, this risk persists after adjusting for traditional $\mathrm{CV}$ risk factors, diabetes duration and diabetes control, suggesting that microvascular disease may contribute to the development of CVD in diabetes. A major finding of the present study is that DR is independently associated with the presence of carotid plaques. This result is similar to that observed in the study by Kreutzenberg et al., in which DR was independently associated with carotid plaques, although the

Table 3 Multivariate logistic regression for the presence of carotid plaques

\begin{tabular}{lll}
\hline & Odds ratio (95\% confidence interval) & P value \\
\hline Age & $1.04(1.01-1.07)$ & 0.0022 \\
BMI & $0.98(0.93-1.03)$ & 0.4578 \\
Female sex & $0.79(0.47-1.34)$ & 0.3952 \\
DR & $1.71(1.03-2.85)$ & 0.0366 \\
Hypertension & $1.78(1.05-3.00)$ & 0.0300 \\
Dyslipidaemia & $1.16(0.71-1.89)$ & 0.5505 \\
Smoking & $2.17(1.13-4.14)$ & 0.0109 \\
eGFR & $1.00(0.99-1.01)$ & 0.3345 \\
UAE & $0.99(0.99-1.00)$ & 0.6397 \\
\hline
\end{tabular}

DR: diabetic retinopathy; UAE: urinary albumin excretion rate; eGFR: estimated glomerular filtration rate. number of patients with retinopathy was lower in that study than in our study, and patients with previous $\mathrm{CV}$ events were not excluded [27]. On the other hand, several studies have described an association between cIMT thickness, as a surrogate marker of atherosclerosis, and DR that is independent of traditional risk factors and glucose control [28,29]. Miyamoto et al. also reported an association between common carotid arterial diameter, as a marker of atherosclerosis, and the presence of DR [30]. It should be noted that in the present study, the percentage of patients with carotid plaques was not only higher in those with DR than in those without DR but that the number of carotid plaques was also elevated. To the best of our knowledge, there is no report in the literature regarding atherosclerotic plaque burden in T2D patients with or without DR. A direct association has been described between a plaque score, consisting of the sum of the maximum plaque thicknesses of different carotid territories, and the presence and extent of coronary artery disease in T2D patients [31]. As reported in general-population studies, the risk

Table 4 Multivariate logistic regression for carotid plaque burden ( $\geq 2$ plaques)

\begin{tabular}{lll}
\hline & Odds ratio (95\% confidence interval) & P value \\
\hline Age & $1.09(1.05-1.13)$ & $<0.0001$ \\
BMI & $0.98(0.92-1.04)$ & 0.6012 \\
Female sex & $0.36(0.19-0.67)$ & 0.0015 \\
DR & $3.17(1.75-5.75)$ & $<0.0001$ \\
Hypertension & $2.00(1.08-3.70)$ & 0.0255 \\
Dyslipidaemia & $2.32(1.33-4.05)$ & 0.0031 \\
Smoking & $1.62(0.77-3.39)$ & 0.1980 \\
eGFR & $1.00(0.99-1.02)$ & 0.3177 \\
UAE & $0.99(0.98-1.00)$ & 0.3107 \\
\hline
\end{tabular}

DR: diabetic retinopathy; UAE: urinary albumin excretion rate; eGFR: estimated glomerular filtration rate. 
of $\mathrm{CV}$ events, such as stroke and cerebral infarction, gradually increases with incremental total plaque number, irrespective of the location of the plaques [32]. Moreover, carotid plaque measurements have been shown to be more strongly predictive of $\mathrm{CV}$ events than cIMT measurements. The extent of atherosclerosis in the carotid artery, measured either as total plaque area [30] or as progression of total plaque volume [12], has been shown to be an independent predictor of coronary events in patients with [12] and without previous CV events [33]. Although these studies included a small proportion of subjects with diabetes, no specific data on these subjects were provided. In the Rotterdam study, a direct relationship between the number of carotid territories with a carotid plaque and incident cases of myocardial infarction was described [34].

Carotid plaque predominantly occurs at sites of nonlaminar turbulent flow, such as the carotid bulb and the proximal ICA, but rarely in the CCA, except in advanced atherosclerotic disease [35]. In the present study, the percentage of plaques in the ICA was higher in T2D patients with DR than in those without DR; however, these differences were not significant. The presence of plaques in the ICA territory is now accepted to be associated with an increased risk of stroke (annual risk of $0.1 \%$ to $1.9 \%$ for an ICA stenosis $<75 \%$ to $80 \%$, and annual risk of $2.0 \%$ to $3 \%$ with greater degrees of stenosis) [36]. Thus, considering research results suggesting that $\mathrm{CV}$ events increase as the burden of atherosclerosis increases, we hypothesise that T2D patients with DR and $>1$ carotid plaque will have a higher risk of $\mathrm{CV}$ events. This speculation can only be answered by conducting a prospective study assessing the future occurrence of CV events in these patients.

As stated in almost all studies on DR, the condition is closely related to duration of the disease, higher blood pressure and a higher albumin excretion rate, all of which may contribute to the increased atherosclerosis in these patients [37]. In the present study, these factors were also more frequent in patients with DR. However, of these variables, only hypertension proved to be independently associated with carotid plaques.

It is now accepted that CKD, defined as an eGFR of $<60 \mathrm{ml} / \mathrm{min}$, is an independent risk factor for atherosclerotic diseases and is associated with the presence of a carotid plaque independent of albuminuria [38]. Recent studies have emphasised that a large number of T2D patients with low eGFR have a normal UAE $[39,40]$. It should be noted that little information regarding renal function has been reported in those studies that analysed subclinical atherosclerosis in patients with T2D. In this respect, the study by Saif et al., conducted inT2D patients with normal renal function, did describe a correlation between DR and cIMT [29].
Another ultrasound measure that is used as a surrogate marker for atherosclerosis is cIMT. This measure, as described for carotid plaques, has been reported to be associated with traditional and newer risk factors for atherosclerosis. The cIMT baseline measures and its progression during follow-up are described to be strongly associated with CVD events, even after adjustment for traditional risk factors [41]. cIMT has typically been measured in the CCA, rather than in the carotid bulb or ICA, because high-level measurement precision is easily obtained from this artery. However, it should be noted that the accuracy of cIMT as a marker of atherosclerosis has been questioned by the fact that medial hypertrophy or intimal thickening of CCA may be influenced by factors that do not necessarily reflect the atherosclerotic process, such as age and hypertension [42]. In the present study, T2D patients with DR had a higher mean-maximum common cIMT than did those without DR, whereas no differences were observed in the bulb-cIMT or in the internal cIMT measures between the groups. However, DR was independently associated with only the mean-internal cIMT. In relation to cIMT, it is accepted that, clinically, the measurement of CCAcIMT is easier and more reliable compared to ICA-cIMT. In contrast, ICA-cIMT appears to predict atherosclerotic cardiac events better than common cIMT [43]. Thus, including internal cIMT and traditional $\mathrm{CV}$ risk factors, as well as the presence of plaque in the internal territory, improves the risk classifications of stroke and coronary heart disease in general population studies.

The association between DR and carotid ultrasound measurements has been analysed in large-population cohort studies conducted on subjects without a history of clinical CVD. The ARIC study found that the severity of retinopathy was associated with carotid artery intimamedia wall thickness [44], while the CHS [45] and MESA [46] studies found no association between DR and cIMT or carotid stenosis. Possible explanations for the different results obtained in these last two studies and ours may be due to the difference in ethnic backgrounds of patient groups, different criteria used for the definition of subclinical atherosclerosis, different population selection or the absence of bulb territory (a site with a high prevalence of plaques) in the ultrasound examination in these studies. In the present study, no differences in cIMT measurements or in plaque presence were observed among the groups with different grades of retinopathy. This finding is in contrast to the findings of Kreutzenberg et al. [27], who described that the retinopathy stage was associated with carotid atherosclerosis. Additionally, in the ARIC study, the severity of retinopathy was associated with cIMT. However, in contrast to the present study, patients with previous $\mathrm{CV}$ events were not excluded in those studies. Moreover, the 
absence of differences observed in cIMT measurements or in plaque presence in relation to retinopathy grades in the present study may be due to few patients in the group having more severe retinopathy. Thus, any conclusion drawn from these results must be made with caution.

In relation to retinopathy and the future risk of $\mathrm{CV}$ events, retinopathy has been described in populationbased samples as a risk factor (independent from diabetes) for stroke [47], heart failure [48] and coronary heart disease [49]. Patients from the ARIC study who had better $\mathrm{CV}$ health, according to the American Heart Association criteria, had a lower prevalence of retinopathy [50]. Moreover, the presence of retinopathy and retinal microvascular signs has recently been described to be associated with an increased risk of cerebral microvascular disease [51].

The strength of the present study is its specific design, which aimed to test the hypothesis that T2D patients with retinopathy have a higher atherosclerotic burden compared with T2D patients without retinopathy. Thus, a large number of patients with retinopathy were included. Only those patients with an eGFR $>60 \mathrm{ml} / \mathrm{min}$ were included to avoid the confounding factor of renal insufficiency, which is associated with an increased risk of atherosclerosis.

The major limitation of the present study is that a direct relationship between retinopathy and macrovascular disease cannot be demonstrated due to the cross-sectional nature of the study. To assess the CV risk associated with a microvascular complication, such as DR in patients with T2D, longitudinal studies are warranted. To properly assess the CV risk, future studies should include T2D patients with DR (with and without carotid plaques) and T2D patients without DR and other microvascular complications.

\section{Conclusions}

The present study, which has been specifically designed to test the hypothesis that T2D patients with DR who are free of previous CVD and have normal renal function have a higher atherosclerotic burden than those without DR, shows an association between DR and subclinical carotid atherosclerosis. More specifically, T2D patients with DR have an increased atherosclerotic burden (presence of plaques and number of plaques) in the carotid territory. Previous studies performed on the general population have shown that individuals with an increased atherosclerotic burden have a higher risk of $\mathrm{CV}$ events (myocardial infarction and stroke). Given the increased atherosclerotic plaque burden observed in the T2D patients with DR in the present study, we believe that these patients represent a target population in which ultrasound examination of the carotid artery should be performed to evaluate the presence/absence of atherosclerotic lesions in this location. In our opinion, given the association between the atherosclerotic plaque burden and the future risk of $\mathrm{CV}$ events shown in generalpopulation studies, a more careful $\mathrm{CV}$ assessment and follow-up should be considered in patients with carotid plaques at ultrasound examination.

\section{Abbreviations}

CCA: Common carotid artery; cIMT: carotid intima-media thickness; CKD: Chronic kidney disease; CV: Cardiovascular; CVD: Cardiovascular disease; DR: Diabetic retinopathy; eGFR: Estimated glomerular filtration rate; ICA: Internal carotid artery; W: Vasa vasorum.

\section{Competing interests}

The authors declare that they have no competing interests.

\section{Authors' contributions}

NA wrote and drafted the manuscript. AT and CJ performed the ophthalmological examination. All of the authors, except for DM, EF and EO, contributed to the data collection. EO participated in the study design, performed the statistical analysis and reviewed the manuscript. ER performed all ultrasound examinations. EF and DM conceived the study, participated in its design and coordination and reviewed the manuscript. All of the authors contributed to the discussion and approved the final manuscript.

\section{Acknowledgements}

This study was supported by grant PS09/01035 from Instituto de Salud Carlos III, Ministry of Economy and Competitiveness, Spain. N. Alcubierre holds a predoctoral fellowship from Instituto de Salud Carlos III FI11/0008.

\section{Author details}

${ }^{1}$ Department of Endocrinology and Nutrition, Health Sciences Research Institute and University Hospital Germans Trias i Pujol, Badalona, Spain. ${ }^{2}$ Department of Ophthalmology, Hospital Universitari Arnau de Vilanova, Lleida, Spain. ${ }^{3}$ Department of Endocrinology and Nutrition, Hospital Universitari Arnau de Vilanova, Lleida, Spain. ${ }^{4}$ Institut de Recerca Biomedica de Lleida, University of Lleida, Lleida, Spain. ${ }^{5}$ Department of Endocrinology and Nutrition, Institut d'Investigacions Biomediques August Pi Suñer, CIBER de Diabetes y Enfermedades Metabólicas asociadas, Hospital Clinic, 08036 Barcelona, Spain. ${ }^{6}$ Unitat de Detecció i Tractament de Malalties

Aterotrombòtiques, Hospital Universitari Arnau de Vilanova, Lleida, Spain. ${ }^{7}$ Department of Neurology, Hospital Universitari Arnau de Vilanova, Lleida, Spain. ${ }^{8}$ Department of Nephrology, Hospital Universitari Arnau de Vilanova, Lleida, Spain.

Received: 11 December 2014 Accepted: 27 February 2015

Published online: 18 March 2015

\section{References}

1. Papa G, Degano C, lurato MP, Licciardello C, Maiorana R, Finocchiaro C. Macrovascular complication phenotypes in type 2 diabetic patients. Cardiovasc Diabetol. 2013;12:20.

2. Kramer CK, Rodrigues TC, Canani LH, Gross JL. Diabetic retinopathy predicts all-cause mortality and cardiovascular events in both type 1 and 2 diabetes: meta-analysis of observational studies. Diabetes Care. 2011;34:1238-44.

3. Cheung N, Wang JJ, Klein R, Couper DJ, Sharrett AR, Wong TY. Diabetic retinopathy and the risk of coronary heart disease: the Atherosclerosis Risk in Communities Study. Diabetes Care. 2007;30:1742-6.

4. Avogaro A, Giorda C, Maggini M, Mannucci E, Raschetti R, Lombardo F, et al. Diabetes and Informatics Study Group, Association of Clinical Diabetologists, Istituto Superiore di Sanità: Incidence of coronary heart disease in type 2 diabetic men and women: impact of microvascular complications, treatment, and geographic location. Diabetes Care. 2007:30:1241-7.

5. Cheung N, Rogers S, Couper DJ, Klein R, Sharrett AR, Wong TY. Is diabetic retinopathy an independent risk factor for ischemic stroke? Stroke. 2007;38:398-401.

6. Jax TW. Metabolic memory: a vascular perspective. Cardiovasc Diabetol. 2010;9:51.

7. Veerman KJ, Venegas-Pino DE, Shi Y, Khan Ml, Gerstein HC, Werstuck GH. Hyperglycaemia is associated with impaired vasa vasorum 
neovascularization and accelerated atherosclerosis in apolipoprotein-E deficient mice. Atherosclerosis. 2013;227:250-8.

8. Orasanu G, Plutzky J. The pathologic continuum of diabetic vascular disease. J Am Coll Cardiol. 2009:53:S35-42.

9. Arcidiacono MV, Traveset A, Rubinat E, Ortega E, Betriu A, Hernández M, et al. Microangiopathy of large artery wall: a neglected complication of diabetes mellitus. Atherosclerosis. 2013;228:142-7.

10. Sampson UK, Harrell FE Jr, Fazio S, Nwosu S, Mercaldo N, Mensah GA, et al. Carotid adventitial Vasa vasorum and Intima-media thickness in a primary prevention population. Echocardiography. 2014. doi:10.1111/echo.12646.

11. Negi SI, Nambi V. The role of carotid intimal thickness and plaque imaging in risk stratification for coronary heart disease. Curr Atheroscler Rep. 2012;14:115-23.

12. Wannarong T, Parraga G, Buchanan D, Fenster A, House AA, Hackam DG, et al. Progression of carotid plaque volume predicts cardiovascular events. Stroke. 2013:44:1859-65.

13. Inaba Y, Chen JA, Bergmann SR. Carotid plaque, compared with carotid intima-media thickness, more accurately predicts coronary artery disease events: a meta-analysis. Atherosclerosis. 2012;220:128-33.

14. Katakami N, Kaneto H, Osonoi T, Saitou M, Takahara M, Sakamoto F, et al. Usefulness of lipoprotein ratios in assessing carotid atherosclerosis in Japanese type 2 diabetic patients. Atherosclerosis. 2011;214:442-7.

15. Bernard S, Sérusclat $A$, Targe $F$, Charrière $S$, Roth $O$, Beaune J, et al. Incremental predictive value of carotid ultrasonography in the assessment of coronary risk in a cohort of asymptomatic type 2 diabetic subjects. Diabetes Care. 2005;28:1158-62.

16. Lau KK, Wong YK, Chan YH, Yiu KH, Teo KC, Li LS, et al. Prognostic implications of surrogate markers of atherosclerosis in low to intermediate risk patients with type 2 diabetes. Cardiovasc Diabetol. 2012;11:101.

17. Kokubo Y, Nakamura S, Okamura T, Yoshimasa Y, Makino H, Watanabe M, et al. Relationship between blood pressure category and incidence of stroke and myocardial infarction in an urban Japanese population with and without chronic kidney disease: the Suita Study. Stroke. 2009;40:2674-9.

18. Choi SW, Kim HY, Lee YH, Ryu SY, Kweon SS, Rhee JA, et al. eGFR is associated with subclinical atherosclerosis independent of albuminuria: the Dong-gu Study. Atherosclerosis. 2010;212:661-7.

19. Yamashita T, Makino H, Nakatani R, Ohata Y, Miyamoto Y, Kishimoto I. Renal insufficiency without albuminuria is associated with peripheral artery atherosclerosis and lipid metabolism disorders in patients with type 2 diabetes. J Atheroscler Thromb. 2013;20:790-7.

20. Ito H, Komatsu Y, Mifune M, Antoku S, Ishida H, Takeuchi Y, et al. The estimated GFR, but not the stage of diabetic nephropathy graded by the urinary albumin excretion, is associated with the carotid intima-media thickness in patients with type 2 diabetes mellitus: a cross-sectional study. Cardiovasc Diabetol. 2010;9:18.

21. Son JW, Jang EH, Kim MK, Kim HL, Baek KH, Song KH, et al. Usefulness of albuminuria as predictor for coronary artery stenosis, regardless of estimated glomerular filtration rate, in patients with type 2 diabetes mellitus. Am J Cardiol. 2012;110:1434-9.

22. Sjöblom P, Nystrom FH, Länne T, Engvall J, Östgren CJ. Microalbuminuria, but not reduced eGFR, is associated with cardiovascular subclinical organ damage in type 2 diabetes. Diabetes Metab. 2014;40:49-55.

23. Wilkinson CP, Ferris 3rd FL, Klein RE, Lee PP, Agardh CD, Davis M, et al. Global Diabetic Retinopathy Project Group. Proposed international clinical diabetic retinopathy and diabetic macular edema disease severity scales. Ophthalmology. 2003;110:1677-82.

24. Levey AS, Bosch JP, Lewis JB, Greene T, Rogers N, Roth D. A more accurate method to estimate glomerular filtration rate from serum creatinine: a new prediction equation. Modification of Diet in Renal Disease Study Group. Ann Intern Med. 1999:130:461-70.

25. Touboul PJ, Hennerici MG, Meairs S, Adams H, Amarenco P, Bornstein N, et al. Mannheim carotid intima-media thickness consensus (2004-2006). An update on behalf of the Advisory Board of the 3rd and 4th Watching the Risk Symposium, 13th and 15th European Stroke Conferences, Mannheim, Germany, 2004, and Brussels, Belgium, 2006. Cerebrovasc Dis. 2007;23:75-80.

26. Rosenson RS, Fioretto P, Dodson PM. Does microvascular disease predict macrovascular events in type 2 diabetes? Atherosclerosis. 2011;218:13-8.

27. de Kreutzenberg SV, Coracina A, Volpi A, Fadini GP, Frigo AC, Guarneri G, et al. Microangiopathy is independently associated with presence, severity and composition of carotid atherosclerosis in type 2 diabetes. Nutr Metab Cardiovasc Dis. 2011;21:286-93.
28. Jung CH, Baek AR, Kim KJ, Kim BY, Kim CH, Kang SK, et al. Association between cardiac autonomic neuropathy, diabetic retinopathy and carotid atherosclerosis in patients with type 2 diabetes. Endocrinol Metab (Seoul). 2013:4:309-19.

29. Saif A, Karawya S, Abdelhamid A. Retinopathy is a strong determinant of atherosclerosis in type 2 diabetes: correlation with carotid intima-media thickness. Endocr Pract. 2014:4:1-21.

30. Miyamoto M, Kotani K, Okada K, Fujii Y, Konno K, Ishibashi S, et al. The correlation of common carotid arterial diameter with atherosclerosis and diabetic retinopathy in patients with type 2 diabetes mellitus. Acta Diabetol. 2012;49:63-8.

31. Akazawa S, Tojikubo M, Nakano Y, Nakamura S, Kawasaki T, Koga N. Usefulness of sum of the thickness of plaque in the carotid artery for predicting the presence and the extent of the coronary artery disease in patients with type 2 diabetes mellitus without known coronary artery disease. Diabetes Res Clin Pract. 2012;96:111-8.

32. Hollander M, Bots ML, Del Sol Al, Koudstaal PJ, Witteman JC, Grobbee DE, et al. Carotid plaques increase the risk of stroke and subtypes of cerebral infarction in asymptomatic elderly: the Rotterdam study. Circulation. 2002;105:2872-7

33. Mathiesen EB, Johnsen SH, Wilsgaard T, Bønaa KH, Løchen ML, Njølstad I. Carotid plaque area and intima-media thickness in prediction of first-ever ischemic stroke: a 10-year follow-up of 6584 men and women: the Troms $\varnothing$ Study. Stroke. 2011;42:972-8.

34. van der Meer IM, Bots ML, Hofman A, del Sol Al, van der Kuip DA, Witteman JC. Predictive value of noninvasive measures of atherosclerosis for incident myocardial infarction: the Rotterdam Study. Circulation. 2004;109:1089-94.

35. O'Leary DH, Bots ML. Imaging of atherosclerosis: carotid intima-media thickness. Eur Heart J. 2010;31:1682-9.

36. Stratton IM, Kohner EM, Aldington SJ, Turner RC, Holman RR, Manley SE, et al. UKPDS 50: risk factors for incidence and progression of retinopathy in Type II diabetes over 6 years from diagnosis. Diabetologia. 2001;44:156-63.

37. Inzitari D, Eliasziw M, Gates P, Sharpe BL, Chan RK, Meldrum HE, et al. The causes and risk of stroke in patients with asymptomatic internal-carotid-artery stenosis. North American Symptomatic Carotid Endarterectomy Trial Collaborators. N Engl J Med. 2000;342:1693-700.

38. Betriu A, Martinez-Alonso M, Arcidiacono MV, Cannata-Andia J, Pascual J, Valdivielso JM, et al. Prevalence of subclinical atheromatosis and associated risk factors in chronic kidney disease: the NEFRONA study. Nephrol Dial Transplant. 2014;29:1415-22.

39. Retnakaran R, Cull CA, Thorne Kl, Adler Al, Holman RR, UKPDS Study Group. Risk factors for renal dysfunction in type 2 diabetes: U.K. Prospective Diabetes Study. Diabetes. 2006;55:1832-9.

40. De Cosmo S, Rossi MC, Pellegrini F, Lucisano G, Bacci S, Gentile S, et al AMD-Annals Study Group. Kidney dysfunction and related cardiovascular risk factors among patients with type 2 diabetes. Nephrol Dial Transplant. 2014;29:657-62.

41. O'Leary DH, Polak JF, Kronmal RA, Manolio TA, Burke GL, Wolfson SK. Carotid-artery intima and media thickness as a risk factor for myocardial infarction and stroke in older adults. Cardiovascular Health Study Collaborative Research Group. N Engl J Med. 1999;340:14-22.

42. Finn AV, Kolodgie FD, Virmani R. Correlation between carotid intimal/medial thickness and atherosclerosis: a point of view from pathology. Arterioscler Thromb Vasc Biol. 2010;30:177-81.

43. Naqvi TZ, Lee MS. Carotid intima-media thickness and plaque in cardiovascular risk assessment. JACC Cardiovasc Imaging. 2014;7:1025-38.

44. Klein R, Sharrett AR, Klein BE, Moss SE, Folsom AR, Wong TY, et al. The association of atherosclerosis, vascular risk factors, and retinopathy in adults with diabetes: the atherosclerosis risk in communities study. Ophthalmology. 2002;109:1225-34.

45. Klein R, Marino EK, Kuller LH, Polak JF, Tracy RP, Gottdiener JS, et al. The relation of atherosclerotic cardiovascular disease to retinopathy in people with diabetes in the Cardiovascular Health Study. Br J Ophthalmol. 2002;86:84-90.

46. Kawasaki R, Cheung N, Islam FM, Klein R, Klein BE, Cotch MF, et al. Multi-Ethnic Study of Atherosclerosis. Is diabetic retinopathy related to subclinical cardiovascular disease? Ophthalmology. 2011;118:860-5.

47. Kawasaki R, Xie J, Cheung N, Lamoureux E, Klein R, Klein BE, et al. MESA: Retinal microvascular signs and risk of stroke: the Multi-Ethnic Study of Atherosclerosis (MESA). Stroke. 2012;43:3245-51.

48. Wong TY, Rosamond W, Chang PP, Couper DJ, Sharrett AR, Hubbard LD, et al. Retinopathy and risk of congestive heart failure. JAMA. 2005;293:63-9. 
49. Wong TY, Klein R, Nieto FJ, Klein BE, Sharrett AR, Meuer SM, et al. Retinal microvascular abnormalities and 10-year cardiovascular mortality: a population-based case-control study. Ophthalmology. 2003;110:933-40.

50. Ogagarue ER, Lutsey PL, Klein R, Klein BE, Folsom AR. Association of ideal cardiovascular health metrics and retinal microvascular findings: the Atherosclerosis Risk in Communities Study. J Am Heart Assoc. 2013;2:e000430

51. Hanff TC, Sharrett AR, Mosley TH, Shibata D, Knopman DS, Klein R, et al. Retinal microvascular abnormalities predict progression of brain microvascular disease: an atherosclerosis risk in communities magnetic resonance imaging study. Stroke. 2014;45:1012-7.

\section{Submit your next manuscript to BioMed Central and take full advantage of:}

- Convenient online submission

- Thorough peer review

- No space constraints or color figure charges

- Immediate publication on acceptance

- Inclusion in PubMed, CAS, Scopus and Google Scholar

- Research which is freely available for redistribution 